\title{
Genetic Dissection of EphA Receptor Signaling Dynamics during Retinotopic Mapping
}

\author{
Nicholas Bevins, ${ }^{1,2}$ Greg Lemke, ${ }^{1}$ and Michaël Reber ${ }^{3}$ \\ ${ }^{1}$ Molecular Neurobiology Laboratory, The Salk Institute, La Jolla, California 92037, ${ }^{2}$ Department of Neurosciences, University of California, San Diego, La \\ Jolla, California 92039, and ${ }^{3}$ INSERM U.575, University of Strasbourg, F-67084 Strasbourg, France
}

Retinal ganglion cells (RGCs) project axons from their cell bodies in the eye to targets in the superior colliculus of the midbrain. The wiring of these axons to their synaptic targets creates an ordered representation, or "map," of retinal space within the brain. Many lines of experiments have demonstrated that the development of this map requires complementary gradients of EphA receptor tyrosine kinases and their ephrin-A ligands, yet basic features of EphA signaling during mapping remain to be resolved. These include the individual roles played by the multiple EphA receptors that make up the retinal EphA gradient. We have developed a set of ratiometric "relative signaling" (RS) rules that quantitatively predict how the composite low-nasal-to-high-temporal EphA gradient is translated into topographic order among RGCs. A key feature of these rules is that the component receptors of the gradient - in the mouse, EphA4, EphA5, and EphA6 must be functionally equivalent and interchangeable. To test this aspect of the model, we generated compound mutant mice in which the periodicity, slope, and receptor composition of the gradient are systematically altered with respect to the levels of EphA4, EphA5, and a closely related receptor, EphA3, that we ectopically express. Analysis of the retinotopic maps of these new mouse mutants establishes the general utility of the RS rules for predicting retinocollicular topography, and demonstrates that individual EphA gene products are approximately equivalent with respect to axon guidance and target selection.

\section{Introduction}

Nervous systems frequently organize representations of the external world into "topographic maps"-systems of synaptic connections in which the positional coordinates of a set of input neurons are maintained in their wiring to synaptic targets (Kaas, 1997; Luo and Flanagan, 2007). The best known and most intensively studied of such maps is the projection of retinal ganglion cells (RGCs) from the vertebrate eye to their synaptic targets in the superior colliculus (SC) (or tectum) of the midbrain (Sperry, 1963; O'Leary and Wilkinson, 1999). This retinocollicular map is Cartesian, in that the orthogonal nasal-temporal (NT) and dorsal-ventral axes of the retina are mapped onto the orthogonal caudal-rostral (CR) and lateral-medial axes, respectively, of the SC (Dräger and Hubel, 1976; Law and Constantine-Paton, 1980; Lemke and Reber, 2005).

Sperry (1963) hypothesized that molecular cues expressed as orthogonal gradients in the retina and the SC provide spatial information that is critical for the development of retinotopic maps, and members of the EphA family of receptor protein ty-

\footnotetext{
Received April 1, 2011; revised May 27, 2011; accepted June 2, 2011

Author contributions: N.B., G.L., and M.R. designed research; N.B. and M.R. performed research; N.B., G.L., and M.R. analyzed data; N.B., G.L., and M.R. wrote the paper.

Acknowledgements: NIH P01NS031249 to G.L. Partnership University Fund to M.R. We thank Todd McLaughlin for invaluable insights, Joe Hash and Patrick Burrola for excellent technical assistance, and the members of the Lemke lab and the Molecular Neurobiology Laboratory for discussion and support.

The authors declare that they have no competing financial interests.

Correspondence should be addressed to either of the following: Greg Lemke, The Salk Institute, 10010 North Torrey Pines Road, La Jolla, CA 92037, E-mail: lemke@salk.edu; or Michaël Reber at his current address: CNRS UPR 3212, University of Strasbourg, F-67084 Strasbourg, France, E-mail: michael.reber@inserm.fr.

DOI:10.1523/JNEUROSCI.1652-11.2011

Copyright $\odot 2011$ the authors $\quad 0270-6474 / 11 / 3110302-09 \$ 15.00 / 0$
}

rosine kinases and their ephrin-A ligands have been found to act as these hypothesized cues (Flanagan and Vanderhaeghen, 1998; Frisén et al., 1998; Feldheim et al., 2000, 2004). The binding of ephrin-As to EphA-receptor-expressing axons is "chemorepulsive," in that it induces actin bundling and axon (and axon branch) retraction (Feldheim et al., 2004). In the retina, RGCs express EphA receptors in a low-nasal-to-high-temporal gradient, and in the SC, ephrin-As are expressed in a low-rostral-tohigh-caudal gradient (Reber et al., 2004; McLaughlin and O'Leary, 2005). Thus, RGCs expressing the highest level of EphA receptors project to the region of the SC with the lowest levels of ephrin-A, and vice versa. All of these observations are consistent with a role for EphA receptors in the development of retinotopy. However, to demonstrate an instructive role for the gradient of EphA receptors and to understand the mode of gradient action, it is necessary to systematically alter the configuration-the slope, orientation, or periodicity - of the gradient without ablating it, and then to systematically measure changes in the configuration of the resulting retinocollicular map.

We have done so, using a line of EphA3 knock-in mice, and a set of mapping rules that we refer to as the "relative signaling" (RS) model (Reber et al., 2004). Together with a precise measurement of the retinal EphA gradient, the RS model quantitatively predicts how the gradient instructs retinotopic map development. We have now generated a set of combined EphA3 knockin/EphA5 knock-out mouse mutants in which the EphA receptor gradient in the retina is altered, but not ablated, and measured the retinocollicular maps of these new mutants. Analysis of these maps confirms the general utility of the RS model as a method for translating EphA gradients into topographic maps. The accuracy 
with which the model predicts topography indicates that EphA3, EphA4, EphA5, and EphA6 are largely interchangeable with respect to their activity during mapping, and demonstrates that the shape and relative magnitude of EphA gradients are essentially informative for the development of retinotopy.

\section{Materials and Methods}

Animal subjects. The Isl2-EphA3 knock-in (Brown et al., 2000), EphA4 knock-out (Dottori et al., 1998), and EphA5 knock-out (Feldheim et al., 2004) mice have all been described previously. The compound mouse mutants used in this paper and in previous analyses are all on hybrid $129 \times \mathrm{C} 57 \mathrm{BL} / 6$ backgrounds. All procedures used in these experiments were reviewed and approved by the Institutional Animal Care and Use Committee at the Salk Institute for Biological Studies. Animals were cared for and used in accordance with guidelines of the U.S. Public Health Service Policy on Humane Care and Use of Laboratory Animals and the NIH Guide for the Care and Use of Laboratory Animals and following institutional Association for Assessment and Accreditation of Laboratory Animal Care-approved practices.

Anterograde labeling. RGC axons were anterogradely labeled by a focal injection of DiI $\left(1,1^{\prime}\right.$-dioctadecyl-3,3,3',3'-tetramethylindocarbocyanine perchlorate) into the retina, as described previously (Brown et al., 2000; Reber et al., 2004), of subject mice of either sex. DiI was dissolved in dimethylformamide, loaded into a pulled glass pipette, and pressure injected into the retina using a Picospritzer. Postnatal day 7 (P7)-P8 mice were injected and the DiI was given $24 \mathrm{~h}$ to migrate along RGC axons, after which time the mice were killed and their SC dissected. The SC was visualized in a wholemount preparation using rhodamine optics on a Zeiss Axioskop 2 upright scope using a $2.5 \times$ objective. The retina was fixed for $48-72 \mathrm{~h}$ in $2 \%$ PFA and then dissected and visualized as a whole-mount preparation.

\section{Results}

\section{The relative signaling (RS) model}

The RS model of topographic mapping is derived from analyses originally performed in the Isl2-EphA3 knock-in mouse (Brown et al., 2000). A subset of mouse RGCs ( $\sim 40 \%)$, distributed evenly throughout the retina (i.e., in approximately every other cell), expresses the transcription factor Islet2 (Isl2) (Pak et al., 2004). In the Isl2-EphA3 knock-ins, EphA3 is produced ectopically and specifically only in these Isl $2{ }^{+}$RGCs (Brown et al., 2000). There are therefore two populations of RGCs that are distinguished by their EphA expression levels: one whose cells express an endogenous level of EphA, and a second whose cells express this endogenous level of EphA plus additional EphA3 (Fig. 1A) (Brown et al., 2000). The target cells of the SC express neither Isl2 nor EphA3. The added level of EphA3 is the same in all Isl2 ${ }^{+}$RGCs, and mice that are homozygous for the Isl2-EphA3 allele express twice as much EphA3 as do Isl2-EphA3 heterozygotes (Reber et al., 2004).

The retinocollicular map of wild-type mice, as visualized using DiI tracing, is a direct, apparently linear transfer of the NT axis of the retina onto the CR axis of the SC (Brown et al., 2000). In marked contrast, Isl2-EphA3 homozygotes (Isl2-EphA3 ${ }^{k i / k i}$ ) display a retinocollicular map that is duplicated across the full NT axis of the retina, in the sense that each point in visual space is represented by two separate points in the SC (Brown et al., 2000; Triplett et al., 2009). A single DiI injection in the retina labels a population of RGCs in the retina consisting of both $\mathrm{Isl} 2^{-}$and adjacent $\mathrm{Isl} 2{ }^{+} / \mathrm{EphA}^{+}{ }^{+}$RGCs. The Isl $2^{+} / \mathrm{EphA}^{+}$RGCs of a given, labeled population project to a distinct, more rostral collicular site-a termination zone or "TZ" — than adjacent, labeled $\mathrm{Isl} 2^{-}$RGCs due to their increased aggregate EphA expression levels and subsequent increased sensitivity to the chemorepulsive CR gradient of ephrin-A in the SC (Fig. $1 B$ ). The preferential occupation of the rostral SC by EphA3 ${ }^{+}$neurons dis-
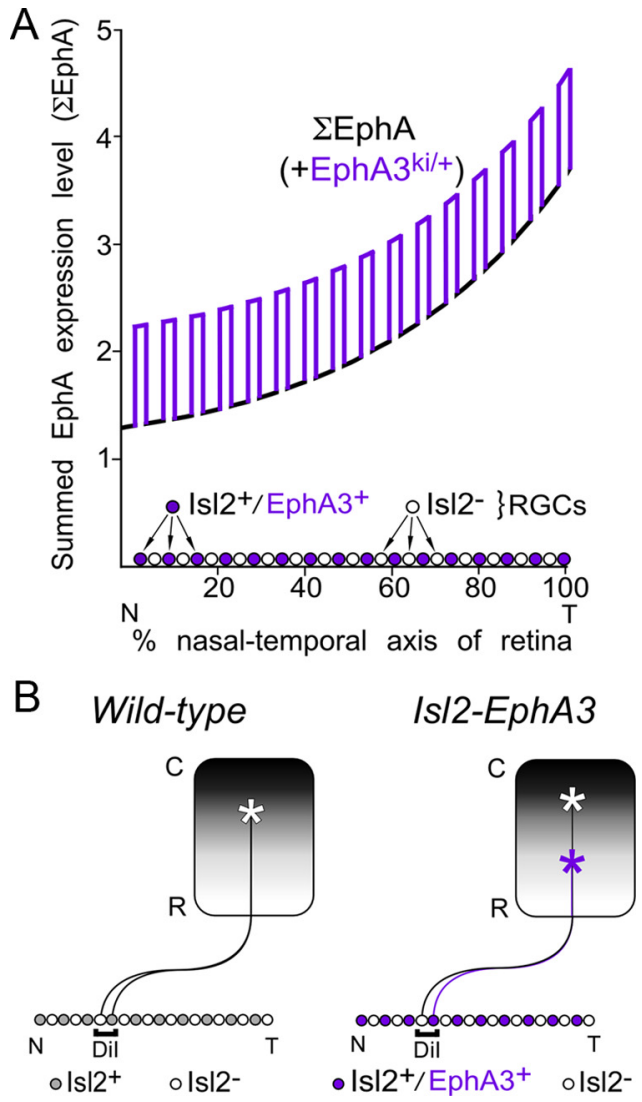

Figure 1. Properties of the $/ s / 2$-EphA3 knock-in mouse. $\boldsymbol{A}$, EphA expression pattern in the retina of $/ s / 2-E p h A 3$ knock-in mice. Approximately every other RGC is either Is 12 expressing (purple circles) or non-IsI2 expressing (white circles) along the nasal-temporal axis of the retina ( $x$-axis). All RGCs express an amount of total EphA ( $\Sigma$ EphA — black line) that increases from nasal to temporal position. IsI2 ${ }^{+} \mathrm{RGC}$ express an additional amount of EphA3 (purple line) that increases the total EphA expressed in these cells. $\boldsymbol{B}$, The effect of ectopic EphA3 expression on retinocollicular mapping. Left, $A$ focal injection of a tracer dye (Dil) into the retina of a wild-type mouse (bracket) labels both $|s| 2^{+}$and $|s| 2^{-}$RGCs. Adjacent IsI2 ${ }^{+}$and $|s| 2^{-}$RGCs expressing the same amount of EphA have the same sensitivity to the high-caudal-to-low-rostral gradient of ephrin-As present in the SC (gray gradient), and map to the same position (TZ) in the SC (white asterisk). Right, A focal Dil injection into the retina of an $/$ s/2-EphA3 knock-in mouse labels both $|s| 2^{+}$and $|s| 2^{-} \mathrm{RGCs}$. IsI $2^{+} \mathrm{RGCs}$ express ectopic EphA3, making these RGCs more sensitive to the ephrin-A gradient in the SC. These $\mathrm{Is} / 2^{+} \mathrm{RGCS}$ therefore map to a more rostral location in the SC (purple asterisk) than their $|\mathrm{s}| 2^{-}$neighbors (white asterisk).

places their wild-type counterparts from these sites, and thereby shifts the entire wild-type map caudally (Brown et al., 2000).

The retinocollicular map of the heterozygous Isl2-EphA3 $\left(I s l 2-E p h A 3^{k i /+}\right)$ mouse exhibits a unique property that is not seen in the Isl2-EphA $3^{k i / k i}$ homozygotes - namely, mapping "collapse" - which has driven both the derivation and analysis of RS mapping rules (Brown et al., 2000; Reber et al., 2004). Like the Isl2-EphA3 $3^{k i / k i}$ mouse, the retinocollicular map of the Isl2EphA $3^{k i /+}$ mouse is duplicated for the nasal-most $76 \%$ of the NT axis (nasal $=0$, temporal $=100$ ), in that a focal DiI injection in the retina labels two TZs in the SC. In contrast to the Isl2$E p h A 3^{k i / k i}$ mouse, however, focal DiI injections in the retina of a Isl2-EphA $3^{k i /+}$ mouse made at positions more temporal than $76 \%$ label only a single TZ in the SC, rather than two (Brown et al., 2000). We refer to this phenomenon as mapping collapse because it occurs suddenly, without a gradual narrowing of the distance between duplicate TZs. We designate the NT position at which collapse occurs as the "collapse point" (Reber et al., 2004); for the Isl2-EphA $3^{k i /+}$ mice, this point is reached at $76 \%$ of the NT 
axis of the retina. The RS model provides a causal explanation for why collapse occurs, and why it occurs in the temporal retina.

The RS model makes use of a precise measurement of the gradient of endogenously expressed EphA receptors in the retina. The EphA family of receptor tyrosine kinases consists of eight proteins (EphA 1-8), which all bind to, and are activated by, any of five ephrin-A ligands (Pasquale, 2005). The endogenous RGC EphA gradient in wild-type mice is composed of an aggregate of the distributions of the three EphA receptors-EphA4, EphA5, and EphA6 (Marcus et al., 2000; Reber et al., 2004). Together with EphA3, these three receptors occupy the same clade within the EphA family. As single receptors, they bind the principal collicular ligands, ephrin-A2 and -A5, with similar, albeit nonidentical affinities (Gale et al., 1996; Monschau et al., 1997). EphA4 is expressed at a constant level across the NT axis of the retina (Fig. $2 \mathrm{~A}$, red curve; equation term in Fig. $2 C$ ), whereas the levels of EphA5 and EphA6 (Fig. 2A, blue and green curves, and equation terms in Fig. $2 C$, respectively) increase smoothly and exponentially from the nasal to the temporal pole (Reber et al., 2004). We have measured these gradients, relative to each other, as mRNA distributions in RGCs across the retina, and have shown that the EphA5 and EphA6 gradients are indeed well fit by exponentials (Reber et al., 2004). The linear summation of EphA4, EphA5, and EphA6 describes the EphA gradient in the retina, and is represented by the term $\Sigma$ EphA. It is important to note that RGC EphA expression levels as a function of position along the NT axis of the retina can only be measured accurately through in situ hybridization-based measurement of mRNAs rather than proteins (Reber et al., 2004). This is due to the fact that EphA proteins are not confined to fixed locations along the NT axis, but are instead transported into RGC axons, all of which course together in the same RGC fiber tract toward the optic disk. This means that a given NT location in the RGC fiber layer always contains axons from RGCs that are positioned more peripherally in the retina. In contrast, EphA mRNAs are for the most part not transported into RGC axons, and remain associated with the ER of RGC cell bodies (Reber et al., 2004). In general, the levels of EphA mRNAs as assessed by in situ hybridization have been found to be well correlated with the levels of EphA proteins as assessed by immunohistochemistry (Cooper et al., 2009).

The two populations of RGCs (Isl2 ${ }^{+}$and $\mathrm{Isl} 2^{-}$) in a knock-in mouse produce two NT gradients of $\Sigma$ EphA: a normal endogenous gradient (Fig. $2 \mathrm{~A}$, black curve), and a second knock-in gradient that includes ectopic EphA3 (Fig. 2 B, purple curve). In the RS model, collapse occurs when the ratiometric difference in EphA signaling between immediately adjacent $\mathrm{Is} 12^{+} / \mathrm{EphA}^{+}$ and Isl2 ${ }^{-} / \mathrm{EphA}^{-}$RGCs is too small for the mapping system to distinguish. In this event, an as-yet-unidentified mapping force causes the adjacent RGCs to map together in the SC, as they would do normally (see Discussion). All Isl ${ }^{+}$RGCs express the same amount of ectopic EphA3; thus, the absolute difference in EphA activity between adjacent $\mathrm{Isl} 2^{+}$and $\mathrm{Isl} 2{ }^{-}$RGCs is the same for adjacent RGCs anywhere in the retina. However, the ratiometric difference in EphA activity between adjacent $\mathrm{Isl}_{2}{ }^{+}$and Isl2 ${ }^{-}$RGCs does change-it decreases from nasal to temporal.

In the RS formalism, the ratiometric difference in EphA signaling between adjacent $\mathrm{Isl} 2^{+} / \mathrm{EphA}^{+}$and $\mathrm{Isl} 2^{-} / \mathrm{EphA}^{-}$ RGCs is designated the local relative signaling ratio $\left(R_{\mathrm{lrs}}\right)$ (Reber et al., 2004). The equation for $R_{\text {lrs }}$ as a function of NT position is given by dividing the $\Sigma$ EphA equation for the knock-in distribution by that for the wild-type distribution (Fig. 2B) (Reber et al., 2004 ). The value of $R_{\text {lrs }}$ below which the system cannot discriminate is the "discrimination limit ratio," and can be directly derived from the $R_{\mathrm{lrs}}$ function and the location of map collapse in Isl2-EphA $3^{k i /+}$ mice: this occurs at $76 \%$ of the NT axis of the retina, where the value of $R_{\text {lrs }}$ is 1.36 (Reber et al., 2004). Thus, the discrimination limit ratio is 1.36 , and when the $R_{\text {lrs }}$ function falls below this value - in any mouse genotype-the retinocollicular map is predicted to collapse.

The $R_{\mathrm{lrs}}$ function can be changed by altering either the slope or magnitude of the underlying $\Sigma$ EphA equation, which can be 
done experimentally either by increasing the dosage of the knock-in allele, as in the Isl2-EphA ${ }^{k i / k i}$ mice, or by crossing this knock-in mouse with knock-out mice for one or more of the EphA genes that contribute to the endogenous, wild-type gradient. The latter manipulation is particularly powerful since $R_{\mathrm{lrs}}$ can be systematically changed in the absence of any change to the absolute level of EphA3 expression. We have performed this manipulation previously by crossing the Isl2-EphA3 knock-in mice with an EphA4 knock-out (Reber et al., 2004). These crosses produce four genotypes of mice, which are either Isl2-EphA $3^{k i / k i}$ or Isl2-EphA $3^{k i /+}$, and at the same time express either half as much EphA4 $\left(E p h A 4^{+/-}\right)$or no EphA4 $\left(E p h A 4^{-/-}\right)$. Each of these genotypes has its own $R_{\text {lrs }}$ function that predicts whether mapping collapse either does or does not occur, and if it does, at what position along the NT axis of the retina collapse is observed ( Reber et al., 2004).

\section{EphA5 tests of the RS model}

These results notwithstanding, several salient features and underlying assumptions of the RS model remain to be assessed. Among the most critical of these is the assumption that all of the EphA receptor genes expressed by RGCs_EphA4, EphA5, EphA6, and in the knock-ins, EphA3 - are functionally interchangeable and equivalent. This very stringent assumption is implicit in the expectation that $\Sigma E$ phA, the summed value of EphA4, EphA5, and EphA6 at each point along the NT axis of the retina, accurately describes the functional properties of the aggregate EphA gradient, and translates directly into differences in aggregate EphA activity during mapping. Indeed, $\Sigma$ EphA has no functional meaning unless EphA3-6 are interchangeable. To alter $\Sigma$ EphA, we have made use of an EphA5 knock-out mouse that carries an in-frame insertion of $\beta$-galactosidase ( $\beta$-gal) that preserves the extracellular, transmembrane, and juxtamembrane domains of the EphA5 protein, but ablates its tyrosine kinase activity (Feldheim et al., 2004; Cooper et al., 2009). We have tested the predictions of the RS model by generating compound mutants of the Isl2-EphA3 knock-in allele and the EphA5 knock-out allele, and measuring their retinocollicular maps. Although the EphA5 mutants are complete knock-outs, these maps should primarily reflect the loss of EphA5 in the retina, since we do not detect significant levels of EphA5 mRNA in the postnatal SC using probes that readily detect expression in the retina (data not shown), and since there is little or no EphA5 mRNA apparent in the SC in the P4 in situ profiles of the online Allen Brain Atlas (http:// developingmouse.brain-map.org/data/Epha5/100072257/thumbnails. html). Previously noted EphA5 protein expression in the SC, as detected by the proxy expression of $\beta$-galactosidase in the EphA5 in-frame $\beta$-gal knock-outs (Feldheim et al., 2004), appears to be due largely to $\beta$-gal expression in afferent RGC axons.

The predictions of the RS rules with regard to EphA5 loss of function are made by modifying the previously described RS equations to reflect the contribution of EphA5 to the endogenous $\Sigma$ EphA gradient. The $R_{\text {lrs }}$ functions for all possible Isl2-EphA3 knock-in/EphA5 knock-out compound mutant mice, based on the measured exponential distribution of EphA5 in the retina (Reber et al., 2004), are displayed in Figure 2D. These functions are generated by dividing the equations that describe the aggregate heterozygous knock-in EphA level $\left(\Sigma E_{p h A}{ }^{k i}\right)$ as a function of NT retinal position in the relevant genotype by the equation that describes aggregate wild-type EphA level ( $\Sigma$ EphA) as a function of NT retinal position in the same genotype (Fig. $2 C$ ). These $R_{\text {lrs }}$ functions predict collapse points at $85 \%$ and $95 \%$ for the
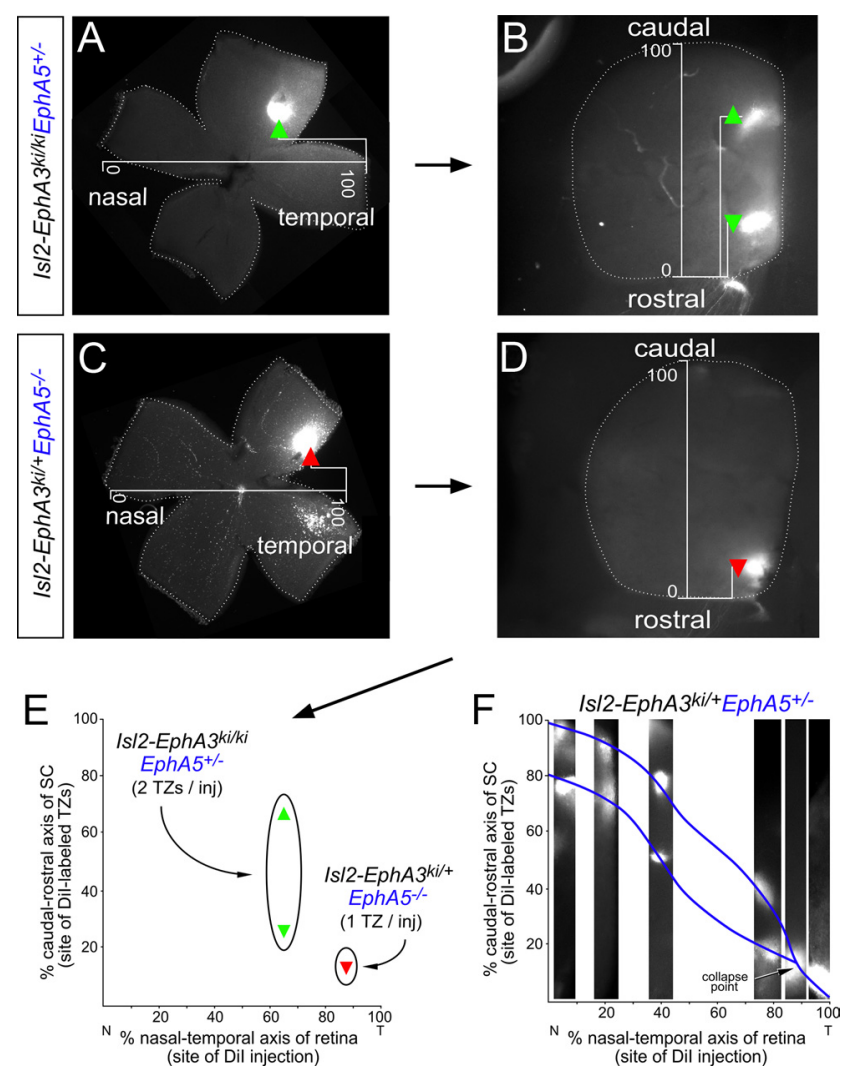

Figure 3. Visualization and measurement of the retinocollicular maps of /s/2-EphA3 knockin/EphA5 knock-out compound mutant mice. $A$, The injection site in the retina is visualized in a whole-mount preparation, an example of which is shown here. The location of the injection site is mapped as percentage of the nasal-temporal axis of the retina, with $0 \%$ being the nasal pole and $100 \%$ being the temporal pole. The SC corresponding to this injected retina is shown in $\boldsymbol{B}$. $\boldsymbol{B}$, The two termination zones of labeled RGC axons in the SC are visualized in a whole-mount preparation, an example of which is shown here. The location of the termination zones is mapped as percentage of the rostral-caudal axis of the SC, with $0 \%$ being the rostral extreme and $100 \%$ being the caudal extreme. This SC corresponds to the retina of $\boldsymbol{A}$ and displays two distinct areas of labeling that are measured as two separate termination zones. Examples in $\boldsymbol{A}$ and $\boldsymbol{B}$ are from an $/ s / 2-E p h A 3^{k i / k i} / E p h A 5^{+/-}$mouse, which is predicted to have a fully duplicated retinocollicular map. $\boldsymbol{C}$, The injection site and retina corresponding to the SC in D. D. The termination zone of the retina seen in $\boldsymbol{C}$. A single, well defined TZ is visible. Examples in $\boldsymbol{C}$ and $\boldsymbol{D}$ are from an $/ s / 2-E p h A 3^{k i /+} / E p h A 5^{-/-}$mouse, which is predicted to have a retinocollicular map that collapses in the temporal retina. $\boldsymbol{E}$, Data points are plotted on a Cartesian graph in which the $x$-axis is the NT position of the retinal Dil injection and the $y$-axis is the CR position of the collicular TZ(s) labeled by this injection. The points plotted in this example correspond to the labeled TZs from $\boldsymbol{B}$ (green) and $\boldsymbol{A}$ (red). Upward-pointing triangles correspond to the more caudal TZs of a duplicated pair; downward-pointing to more rostral. $\boldsymbol{F}$, A map for any given genotype is made by plotting multiple map points, obtained from systematic Dil injections across the full NT extent of the retina, from mice of the same genotype. Example sections of the $S C$ of multiple animals are shown at the position of the $x$-axis corresponding to the location of their respective Dil injections in the retina (retinas not shown). The blue line connects the data points and represents the continuity of the map in vivo. The example points shown here correspond to the $/ s / 2-E p h A 3^{k i /+} / E p h A 5^{+/-}$genotype, a complete map of which is shown in Figure $4 A$.

Isl2-EphA $3^{k i /+} / E p h A 5^{+/-}$and Isl2-EphA $3^{k i /+} / E p h A 5^{-/-}$mice, respectively, based on where the $R_{\mathrm{lrs}}$ function crosses the discrimination limit ratio (Fig. 2D). The $R_{\text {lrs }}$ functions for the Isl2$E p h A 3^{k i / k / i} / E p h A 5^{+/-}$and Isl2-EphA3 $3^{k i / k i} / E p h A 5^{-1-}$ mice predict noncollapsing maps, since these $R_{\mathrm{lrs}}$ curves do not cross the discrimination limit ratio (Fig. 2D).

We measured the retinocollicular maps of these mice anatomically, using focal retinal injections of DiI as described previously (Brown et al., 2000; Reber et al., 2004). DiI injected into the retina (Fig. $3 \mathrm{~A}, \mathrm{C}$, retinal flat mount examples) diffuses down the axons 
of RGCs into the SC and becomes concentrated at synaptic TZs, where it is readily visualized in collicular whole mounts $24 \mathrm{~h}$ after injection (examples in Fig. $3 B, D$ ). An example of a duplicated TZ (two TZs from a single retinal DiI injection) is shown in Figure $3 B$ and an example of a single TZ (one TZ from a single retinal DiI injection) is shown in Figure $3 D$. The location site of the injection and the corresponding TZ(s) are plotted as points on a Cartesian graph, where the $x$-axis is the NT axis of the retina and the $y$-axis is the CR axis of the SC (example in Fig. $3 E$ ). To visualize the entire retinocollicular map of any given genotype, we plot data points from multiple injections performed systematically across the full NT retinal axis in multiple mice of that genotype. Careful measurement of the NT axial position of the injection site and the $\mathrm{CR}$ axial position of the collicular TZ(s) that are labeled by these injections allows for a delineation of the entire retinocollicular map for a given genotype (example in Fig. $3 F$ ).

The RS model makes two precise predictions about the compound Isl2-EphA3 knock-in/EphA5 knock-out mutants: whether the map will be fully duplicated or not, and, if the map is not fully duplicated, at what point along the NT axis of the retina the map will collapse from duplicated to single. Testing the first prediction is a straightforward matter of observing whether the map collapses. Testing the second prediction requires a precise measurement of the location on the NT axis of the retina where collapse is seen. We have used the locations of the lateral and medial rectus muscles, on the exterior of the eye globe, to determine the nasal and temporal poles of the retina, respectively. Mice do not have an appreciable fovea (Jeon et al., 1998), or any other landmark, within the retina to determine the orientation of the location of the nasal and temporal poles after dissection. Thus, we must rely on the accuracy of our dissections to determine the location of the injection site within the retina, with the acknowledgment that there is some error in this measurement (and that any attempt to precisely quantify that error would be speculative).

The Isl2-EphA $3^{k i /+} / E p h A 5^{+/-}$and Isl2-EphA3 $3^{k i /+} /$ EphA $^{-/-}$ maps that we measured using these methods are shown in Figure 4, $A$ and $B$, respectively. As predicted (Fig. $2 D$, solid curves), these two maps are very similar, and both display the phenomenon of mapping collapse in the temporal retina. In addition, their collapse points are situated at approximate positions along the NT axis- $85 \%$ and $90 \%$, respectively - that are very close to the location predicted by the point at which the $R_{\mathrm{lrs}}$ functions of Figure $2 D$ cross the discrimination limit ratio.

The RS rules predict that all compound mutants of Isl2$E p h A 3^{k i / k i}$ homozygous knock-ins and any combination of EphA4, EphA5, or EphA6 knock-outs, either heterozygous or homozygous, should all exhibit fully duplicated, noncollapsing retinocollicular maps that are largely indistinguishable from each other. This is due to the fact that the Isl2-EphA $3^{k i / k i}$ homozygous knock-ins already display a fully duplicated map (Triplett et al., 2009), and all reductions in endogenous EphA levels that are generated by crossing the knock-ins with any EphA knockouts result in increases in $R_{\text {lrs }}$. The measured Isl2-EphA3 ${ }^{k i / k i} /$ EphA $5^{+/-}$and Isl2-EphA $3^{k i / k i} / E p h A 5^{-1-}$ maps are shown in Figure 4, $C$ and $D$, respectively. As predicted (Fig. 2D), both genotypes show fully duplicated, noncollapsing maps.

\section{Relative signaling also predicts mapping density}

The Isl2-EphA3 $3^{k i /+} / E p h A 5^{+/-}, / E p h A 5^{-1-}, / E p h A 4^{+/-}$, and IEphA4 ${ }^{-/-}$compound mutant mice all display retinocollicular maps that collapse, with collapse points that closely match those predicted by the RS rules. However, there is a striking difference in the extent of rostral-caudal separation of duplicated collicular
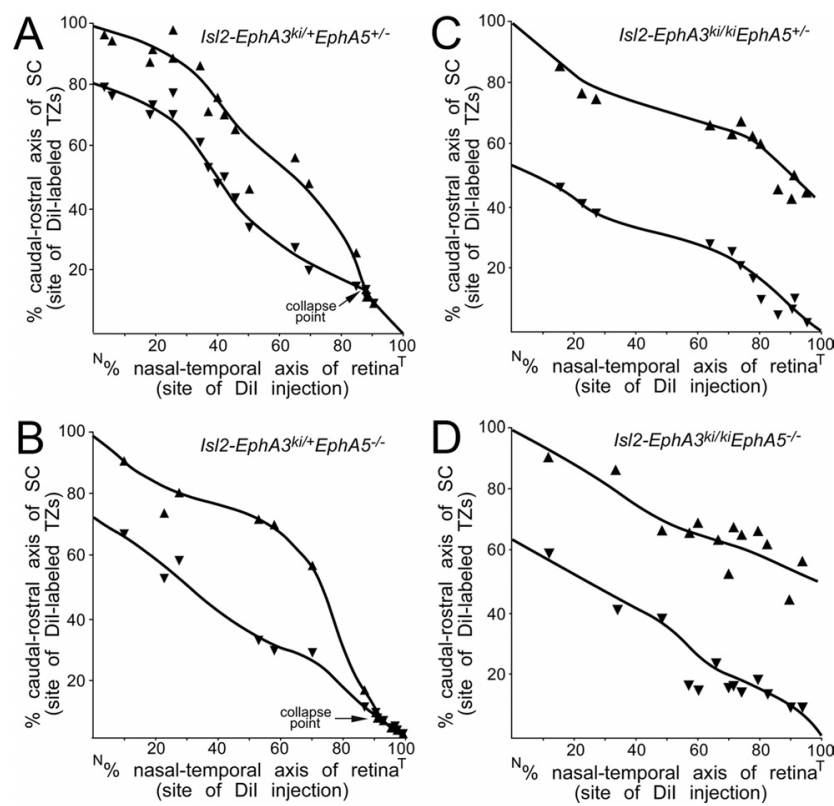

Figure 4. Anterograde labeling of /s/2-EphA3 knock-in, EphA5 knock-out compound mutant retinocollicular maps. $A$, Retinocollicular map of $I s / 2-E p h A 3^{k i /+} / E p h A 5^{+/-}$compound mutant. The position of the injection site in the retina is graphed on the $x$-axis. The position of $T Z(s)$ in the $S C$ is graphed on the $y$-axis. Each pair of points corresponds to a single retinal injection in a single mouse. The upward-pointing triangles correspond to the caudal TZ in an animal with two TZs. The downward-pointing triangles correspond to the rostral TZ in an animal with two TZs. The hourglass shapes correspond to a collapsed TZ in an animal showing a single TZ. The collapse point is located at $\sim 85 \%$ of the NT axis, which is at the predicted position. B, Retinocollicular map of Is/2-EphA3 $3^{k i /} /$ EphA5 $5^{-/-}$compound mutant. As predicted, the map collapses. The collapse point is located at $\sim 90 \%$ of the NT axis, which is very close to the predicted position. C, Retinocollicular map of $/ s / 2-E p h A 3^{k i / k i} / E p h A 5^{+/-}$compound mutant. As predicted, the map is fully duplicated. $\boldsymbol{D}$, Retinocollicular map of $/ \mathrm{s} / 2-E p h A 3^{k i / k i} / E p h A 5^{-1-}$ compound mutant. As predicted, the map is fully duplicated.

TZs between the EphA4 and EphA5 loss-of-function compound mutants. TZ separation in the Isl2-EphA3 $3^{k i /+} / E p h A 5^{+/-}$compound mutants is $\sim 20 \%$ of the collicular CR axis (Fig. $4 A$ ) - an axial separation that is very similar to that seen in the Isl2$E p h A 3^{k i /+}$ knock-ins alone (Brown et al., 2000). The average TZ separation in the Isl2-EphA $3^{k i /+} / E p h A 5^{-/-}$mice ( $\sim 30 \%$; Fig. $4 B)$ is only slightly greater than that of the $I s l 2-E p h A 3^{k i /+}$ / $E p h A 5^{+/-}$mice. In marked contrast, TZ separation in the Isl2$E p h A 3^{k i /+} / E p h A 4^{+/-}$and /EphA $4^{-1-}$ mice is much larger$>50 \%$ of the collicular CR axis (Reber et al., 2004) (Fig. 5A).

In all of the 10 different genotypes of knock-in mice that we have analyzed, the number of Isl $2{ }^{+}$RGCs is constant, and these Isl2 ${ }^{+}$RGCs always map to the rostral portion of the SC. The rostral-caudal separation of TZs is therefore a direct measurement of the density of Isl $2{ }^{+}$RGCs in this portion of the SC (Fig. $5 A$ ). Under the ratiometric RS formalism, the mapping density, the number RGCs mapping within a given SC area, of Isl $2{ }^{+} \mathrm{RGCs}$ should be determined by the total amount of EphA expressed by Isl2 ${ }^{+}$RGCs relative to the total amount of EphA expressed by Isl2 ${ }^{-}$RGCs. This ratio, which we refer to as the population relative signaling ratio $\left(R_{\mathrm{prs}}\right)$, is analogous to the $R_{\mathrm{lrs}}$ described previously, but is a function of the total amount of EphA expressed by all RGCs of a given type, Isl $2^{+}$or Isl2 ${ }^{-}$. Given our measurement of the EphA receptor expression levels in RGCs, $R_{\text {prs }}$ can be calculated precisely for any compound mutant mouse using the following steps. The total amount of EphA expressed by a given population of RGCs $\left(\mathrm{Isl}_{2}{ }^{+}\right.$or Isl2 ${ }^{-}$) is calculated by integrating the $\Sigma$ EphA function for that RGC population across the entire 


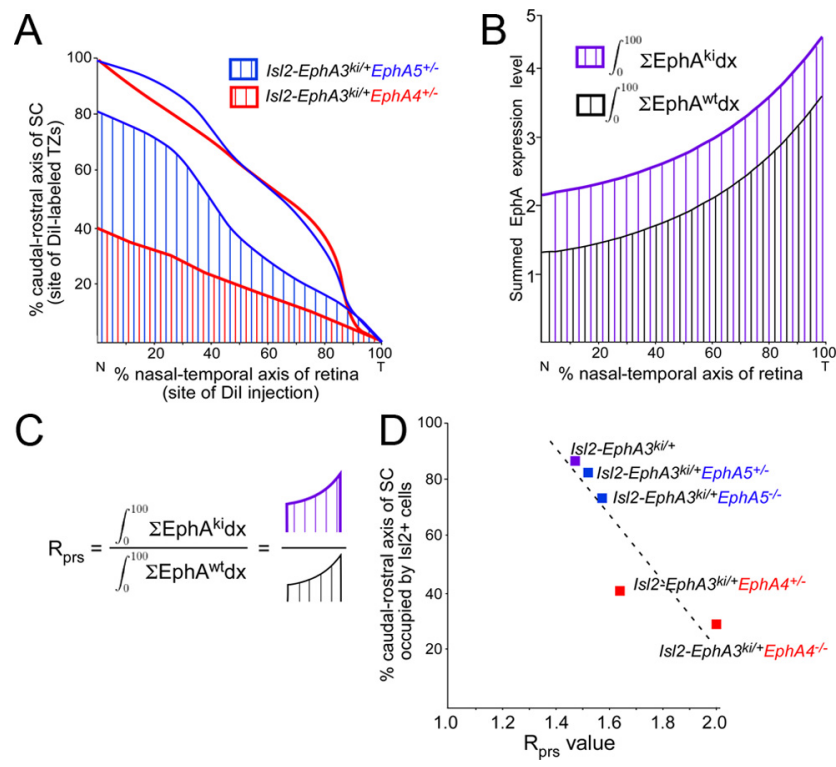

Figure 5. Population relative signaling ratio and mapping density. $\boldsymbol{A}$, The retinocollicular maps for mice of the $I s / 2-E p h A 3^{k i /+} / E p h A 5^{+/-}$genotype (blue lines) and $I s / 2-E p h A 3^{k / /+} /$ $E p h A 4^{+/-}$genotype (red lines) (Reber et al., 2004) are shown. The lower line of each map corresponds to the location of $\mathrm{Is} / 2{ }^{+} \mathrm{RGCS}$. The area covered with vertical lines shows the area of the SC where IsI2 ${ }^{+}$RGCs map in $/ s / 2-E p h A 3^{k i /+} / E p h A 5^{+/-}$mice (blue lines) and $/ s / 2-E p h A 3^{k i /+}$ / $E p h A 4^{+/-}$mice (red lines). $\boldsymbol{B}$, The total amount of EphA expressed by a population of RGCs can be calculated by integrating the function for $\Sigma$ EphA for that population of RGCs for $x=0$ to $x=$ 100 , which corresponds to the entire NT axis of the retina. The integral for $\Sigma E p h A{ }^{k i}$ (purple) will always be larger than that for $\sum$ EphA (black) in a knock-in mouse. $C$, The population relative signaling ratio $R_{\text {prs }}$ is calculated for any given genotype by dividing the integral of the $\sum E$ Eph ${ }^{\mathrm{ki}}$ function by the integral of the $\Sigma$ EphA relevant for that genotype. $D$, The $R_{\text {prs }}$ values for $I s / 2$ $E p h A 3^{k i /+}$ compound mutants ( $x$-axis) and the percentage of the SC occupied by Isl2 ${ }^{+} \mathrm{RGCS}$ ( $y$-axis) are shown.

NT axis of the retina (Fig. $5 B$ ). The $R_{\text {prs }}$ is then calculated by dividing the integral of the $\Sigma$ EphA for Isl2 ${ }^{+}$RGCs by the integral of the $\Sigma$ EphA function for Isl ${ }^{-}$RGCs (Fig. $5 C$ ). The $R_{\text {prs }}$ values for all Isl2-EphA3 knock-in, and for EphA4 or EphA5 knock-out compound mutant genotypes, are plotted on the $x$-axis of Figure $5 D$, with the total area of the SC occupied by Isl $2^{+}$RGCs plotted on the $y$-axis. It is clear that the area of the SC occupied by Isl $2^{+}$ RGCs is inversely proportional to the $R_{\text {prs }}$ value (Fig. 5D), which is consistent with the chemorepulsive action of EphA-ephrin-A forward signaling.

In all maps that collapse, Isl $2^{-}$RGCs from the temporal retina are "pulled" into the rostral SC, causing them to map onto the entire rostral-caudal extent of the SC. The density of Isl2 ${ }^{-}$RGCs is therefore the same for all genotypes in collapsed maps. The Isl2 ${ }^{-}$RGCs that move into the rostral SC open up space in more caudal areas, where Isl2 ${ }^{+}$RGCs can then map. Mapping collapse causes the density of RGCs in the SC to become heterogeneous, and the extent to which Isl2 ${ }^{+}$RGCs move into the caudal colliculus is proportional to the value of $R_{\text {prs }}$. Without mapping collapse, the density of RGC mapping will be homogeneous throughout the SC. The density of $\mathrm{Isl}_{2}{ }^{+}$and $\mathrm{Isl} 2^{-}$ RGCs is therefore always the same in maps that are fully duplicated, despite dramatic differences in $R_{\text {prs }}$ values between genotypes. In WT maps, the mapping density is homogeneous because the $R_{\text {prs }}$ value is 1 .

\section{The EphA5 $5^{-1-}$ retinocollicular map}

The RS rules predict that the retinocollicular maps of homozygous EphA4, EphA5, or EphA6 knock-out mice should be very
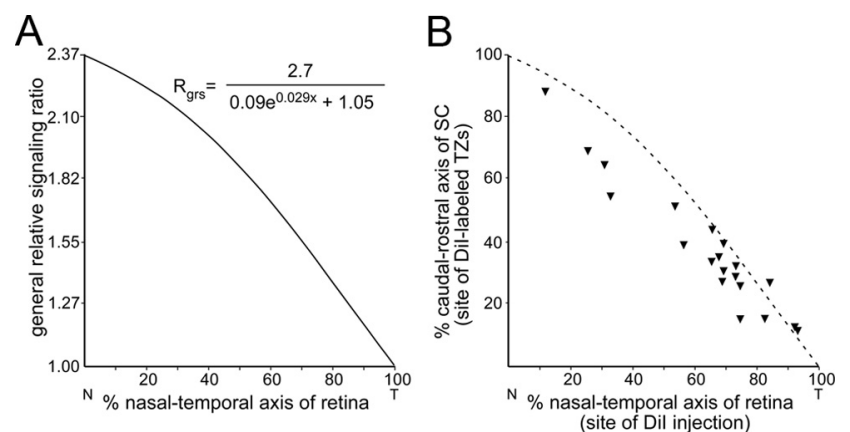

Figure 6. General relative signaling prediction of the $E p h A 5^{-/-}$map. $\boldsymbol{A}$, The general relative signaling ratio $\left(R_{\text {grs }}\right)$ function is calculated by dividing the $\Sigma$ EphA value at the temporal pole of the retina by the $\Sigma$ EphA value at position $x$. The $R_{\text {grs }}$ function of $E p h A 5^{-1-}$ mice is shown. The value of the $R_{\text {grs }}$ is plotted on the $y$-axis for each position along the NT $(x)$ axis of the retina. $\boldsymbol{B}$, The EphA5 ${ }^{-1-}$ retinocollicular map, as determined by repeated Dil injections across the NT axis of the retina, is shown (blue triangles) superimposed on the $R_{\text {grs }}$ function (dashed blue line).

similar to the map of wild-type mice (Reber et al., 2004); that is, there should be no appreciable effect on the configuration of the map from removing these individual receptors. This prediction, which has never been tested rigorously, arises from the application of "general relative signaling" rules, which are a straightforward extension of the local relative signaling rules (Reber et al., 2004). We have previously demonstrated that the application of general relative signaling yields the wild-type retinocollicular map in the mouse (Reber et al., 2004). In the general RS formalism, the configuration of the wild-type mouse retinocollicular map is predicted, with remarkable accuracy, simply by dividing the measured $\Sigma$ EphA value at the extreme temporal pole of the retina $\left(\Sigma E p h A_{t}\right)$ by the equation that specifies $\Sigma E$ phA as a function of position across the NT axis of the retina (Reber et al., 2004). This operation yields the $\Sigma$ EphA ratio between the temporal pole RGC and all other RGCs across this axis, and is designated the general RS ratio $\left(R_{\mathrm{grs}}\right)$. This general RS ratio is based on "competition by exclusion" (Reber et al., 2004), in which the temporal-most RGC, which expresses the highest level of $\Sigma$ EphA and is therefore most sensitive to the collicular ephrin-A gradient, sets the rostral-most mapping point and drives mapping. Note that the local relative signaling ratio for immediately adjacent RGCs $\left(R_{\mathrm{lrs}}\right)$ is derived simply by dividing their respective $R_{\mathrm{grs}}$ values (Reber et al., 2004). We asked whether a general RS rule would predict the retinocollicular map in EphA $5^{-1-}$ mice, which retain expression of the EphA4 and EphA6 components of the endogenous $\Sigma$ EphA gradient.

The general RS ratio $\left(R_{\mathrm{grs}}\right)$ equation for $E p h A 5^{-1-}$ mice, which lack the graded EphA5 component of the composite $\Sigma$ EphA gradient, is indicated and plotted in Figure $6 \mathrm{~A}$. This plot is very similar to both the predicted and measured wild-type retinocollicular map (Reber et al., 2004). The EphA5 ${ }^{-1-}$ retinocollicular map that we determined by repeated DiI injections is illustrated in Figure $6 \mathrm{~B}$. With the exception of the nasal third of the retina, where there is a slight rostral deviation from prediction, this measured EphA5 $5^{-1-}$ map is in very close agreement with the EphA5 ${ }^{-1-}$ map predicted by general relative signaling.

\section{Discussion}

Requirement of a specific EphA gradient for the development of retinotopy

Sperry's chemoaffinity hypothesis has been a guiding principle of the study of neural development since its inception (Sperry, 
1963). Genetic and molecular experiments have clearly demonstrated that for mapping of the NT axis of the retina onto the CR axis of the SC, the EphA receptors and collicular ephrin-A ligands act as the molecular cues of Sperry's hypothesis (Flanagan and Vanderhaeghen, 1998; Brown et al., 2000). However, to specify position with such a limited number of molecular cues, the developing nervous system must use the information contained within the gradient of gene expression levels (Gierer, 1988). A formal test of this hypothesis requires a quantitative model of the role that gradients play in topographic mapping, followed by tests of the model's predictions. Relative signaling (Reber et al., 2004) is such a model, and we here report its ability to predict topography across multiple novel mouse EphA genotypes.

The rules of the RS model are based on the principle that topographic order is established through ratiometric differences in $\Sigma$ EphA receptor expression and signaling activity among RGCs. These ratiometric differences may be manipulated experimentally. As detailed above, by removing half or all of the graded EphA5 receptor in the Isl2-EphA3/EphA5 compound mutants, we have altered both the periodicity and the slope of the $\Sigma E$ phA gradient. Our earlier tests of the RS model altered the periodicity and the magnitude of the $\Sigma$ EphA receptor gradient, by removing half or all of ungraded EphA4 in the Isl2 EphA3/EphA4 compound mutants (Reber et al., 2004). These different manipulations lead to distinct sets of RS ratios, yet in all settings, it is these ratios that set the configuration of the retinocollicular map.

\section{EphA signaling during development}

The robustness of the RS rules across multiple EphA3, EphA4, and EphA5 mutant mouse genotypes supports the implicit assumption that the activities of these proteins are equivalent with respect to the events of retinocollicular mapping. As noted above, this assumption is reflected in the summation of EphA receptor activity to yield EphA and in the predictions made by ratiometric RS difference comparisons in EphA (rather than individual EphA receptors). Although the measured binding affinities of EphA3, EphA4, EphA5, and EphA6 for ephrin-A2 and ephrin-A5 are not identical (Gale et al., 1996; Monschau et al., 1997), these measurements were generally made in settings in which the binding of artificially clustered single ephrin-As were monitored against a single EphA receptor. This is not the case in vivo, where multiple EphAs typically signal as exceptionally large, multimeric complexes that often interact with multiple ephrin-As (Palmer and Klein, 2003). It is therefore possible that the observed equivalence of EphAs in retinocollicular mapping might reflect signaling complex averaging of differences in individual EphA-ephrin-A affinities. It should be noted that the interchangeability of EphAs with respect to retinocollicular mapping is clearly evident in evolution. The principal graded EphA receptor that contributes to the RGC gradient in the chick, where the EphA gradient was first discovered, is EphA3 (Cheng et al., 1995). This receptor is not even expressed by mouse RGCs (Brown et al., 2000), which instead substitute EphA5 and EphA6. The phenomenon of EphA equivalence in retinocollicular mapping may have general relevance, since EphA receptors guide cytoskeletal rearrangements in many additional biological contexts outside of the nervous system (Pasquale, 2005).

Previous tests of the RS model relied on the addition or removal of a complete EphA gene or cDNA. In the experiments described above, we instead used an EphA5 mutant in which the intracellular kinase domain of EphA5 is replaced by lacZ, which yields RGCs that produce an EphA5 protein that retains its ability to bind to ligands but is kinase-dead (Feldheim et al., 2004). $\beta$-Galactosidase staining is evident in the optic tract of these EphA5-lacZ mutant mice at postnatal day 5 (our unpublished data; Cooper et al., 2009), suggesting that RGC localization of mutant EphA5 is similar to that of wild type. The fact that the predictions of the RS model are valid in these mice is consistent with the hypothesis that $\Sigma$ EphA represents both the summed expression level and the summed kinase activity of its component EphA receptors.

\section{Competition/relative signaling as a mapping constraint}

The RS model integrates competition as a constraint based on previous theoretical considerations of retinocollicular mapping. Experiments showing map compression, in which part of the SC is removed before development of retinotopy and all of the RGCs are compressed into the remaining SC (Sharma, 1972), as well as map expansion, in which part of the retina is removed and the remaining RGCs expand their map to cover the entire SC (Schmidt and Easter, 1978), demonstrated that the mapping process requires an element of competition among RGCs for a limiting factor present in the SC (Prestige and Willshaw, 1975). Likewise, for compression and expansion of the map to occur, RGCs must read their molecular cues relative to other RGCs. Competition is evident in the mapping behavior of Isl2-EphA3 homozygotes alone. In these mice, the TZs of all wild-type RGCs are pushed caudally from their normal mapping position by their Isl $2^{+} /$EphA $^{+}$neighbors, despite the fact that the $\Sigma$ EphA profile of the wild-type RGCs is unchanged, i.e., entirely normal (Brown et al., 2000).

Many models of retinocollicular mapping have posited that competition between RGCs for a limiting factor in the SC (Goodhill and $\mathrm{Xu}, 2005$ ) balances the unidirectional force of EphA-ephrin-A forward signaling, but a molecular basis for competition in mapping has not been established. We present an extension of the RS model that describes the mapping density of a population of RGCs in the SC-the population relative signaling ratio $\left(R_{\mathrm{prs}}\right)$ - and that also reflects competition. Calculation of the $R_{\text {prs }}$ involves an arithmetic that is very similar to that of the $R_{\text {lrs }}$, but applies this arithmetic to a population of RGCs rather than to a pair of RGCs. The ability of the $R_{\text {prs }}$ ratio to reconcile mapping density and population EphA expression levels suggests that changes in mapping density are a manifestation of competition.

A final indicator of opponent activities is the sudden mapping collapse that we consistently see across multiple compound genotypes that are heterozygous for the Isl2-EphA3 allele. At the point at which mapping collapse occurs, adjacent Isl $2^{+} / \mathrm{EphA}^{+}$ and Isl2 ${ }^{-} / \mathrm{EphA}^{-}$RGCs are subject to two opponent effects: a disparity in EphA that pushes these RGCs apart with respect to their termination in the SC, and a correlation in the pattern of their firing that brings their TZs together (Butts, 2002). When the ratiometric difference in EphA is too low for the mapping system to discriminate, the effect of correlated activity is suddenly revealed-causing collapse. Correlated electrical firing is well known for its ability to refine and consolidate retinocollicular TZs (McLaughlin et al., 2003; Chandrasekaran et al., 2005; MrsicFlogel et al., 2005; Cang et al., 2008), although the molecules that underlie this activity remain a focus of study.

\section{Comparison of the RS model with in silico considerations of retinocollicular mapping}

Models of biological phenomena may be more informative than direct observation of experimental data alone. Computational 
models highlight parts of a complex process to make it more easily understood, but must, at the same time, also ignore or overly simplify other parts of that same process. A model is, by necessity, "a simplification and an idealization, and consequently a falsification” (Turing, 1952, p 37). A complete model of retinotopic map formation requires greater detail than the RS model (Goodhill and Richards, 1999), and many computational models attempt to model the process in its entirety (Goodhill, 2007). These models are usually too complex to be directly tested by tractable in vivo experiments and are instead tested in silico. In fact, these models are often tested by their ability to recreate the altered topography in Isl2-EphA3 knock-in animals reported by Brown et al. (2000) and Reber et al. (2004), and can also be tested using the data we report here (Honda, 2003; Koulakov and Tsigankov, 2004; Simpson and Goodhill, 2011; Willshaw, 2006). The RS model provides useful information that would not be apparent without computation, while at the same time, it makes concrete, prospective, testable predictions as to the configuration of the map in genetically manipulated animals that grounds abstraction in experimental data.

\section{General applications of the relative signaling model}

The cellular mechanisms of map formation that we infer from the utility of the RS model, such as the aggregate signaling and interchangeability of EphA receptors, may also be important for the development of topography in other locations where EphA receptors are expressed as gradients. These include the olfactory bulb (Cutforth et al., 2003), the projections of motor neurons to muscles (Helmbacher et al., 2000; Eberhart et al., 2002; Kania and Jessell, 2003), and the hippocampal-septal projection (Gao et al., 1996). Indeed, many aspects of neural development are similar, generally, to those of retinotopic mapping, and may be governed by relative signaling rules comparable to those we describe.

\section{References}

Brown A, Yates PA, Burrola P, Ortuño D, Vaidya A, Jessell TM, Pfaff SL, O'Leary DD, Lemke G (2000) Topographic mapping from the retina to the midbrain is controlled by relative but not absolute levels of EphA receptor signaling. Cell 102:77-88.

Butts DA (2002) Retinal waves: implications for synaptic learning rules during development. Neuroscientist 8:243-253.

Cang J, Wang L, Stryker MP, Feldheim DA (2008) Roles of ephrin-As and structured activity in the development of functional maps in the superior colliculus. J Neurosci 28:11015-11023.

Chandrasekaran AR, Plas DT, Gonzalez E, Crair MC (2005) Evidence for an instructive role of retinal activity in retinotopic map refinement in the superior colliculus of the mouse. J Neurosci 25:6929-6938.

Cheng HJ, Nakamoto M, Bergemann AD, Flanagan JG (1995) Complementary gradients in expression and binding of ELF-1 and Mek4 in development of the topographic retinotectal projection map. Cell 82:371-381.

Cooper MA, Crockett DP, Nowakowski RS, Gale NW, Zhou R (2009) Distribution of EphA5 receptor protein in the developing and adult mouse nervous system. J Comp Neurol 514:310-328.

Cutforth T, Moring L, Mendelsohn M, Nemes A, Shah NM, Kim MM, Frisén J, Axel R (2003) Axonal ephrin-As and odorant receptors: coordinate determination of the olfactory sensory map. Cell 114:311-322.

Dottori M, Hartley L, Galea M, Paxinos G, Polizzotto M, Kilpatrick T, Bartlett PF, Murphy M, Köntgen F, Boyd AW (1998) EphA4 (Sek1) receptor tyrosine kinase is required for the development of the corticospinal tract. Proc Natl Acad Sci U S A 95:13248-13253.

Dräger UC, Hubel DH (1976) Topography of visual and somatosensory projections to mouse superior colliculus. J Neurophysiol 39:91-101.

Eberhart J, Swartz ME, Koblar SA, Pasquale EB, Krull CE (2002) EphA4 constitutes a population-specific guidance cue for motor neurons. Dev Biol 247:89-101.

Feldheim DA, Kim YI, Bergemann AD, Frisén J, Barbacid M, Flanagan JG (2000) Genetic analysis of ephrin-A2 and ephrin-A5 shows their require- ment in multiple aspects of retinocollicular mapping. Neuron 25:563-574.

Feldheim DA, Nakamoto M, Osterfield M, Gale NW, DeChiara TM, Rohatgi R, Yancopoulos GD, Flanagan JG (2004) Loss-of-function analysis of EphA receptors in retinotectal mapping. J Neurosci 24:2542-2550.

Flanagan JG, Vanderhaeghen P (1998) The ephrins and Eph receptors in neural development. Annu Rev Neurosci 21:309-345.

Frisén J, Yates PA, McLaughlin T, Friedman GC, O’Leary DD, Barbacid M (1998) Ephrin-A5 (AL-1/RAGS) is essential for proper retinal axon guidance and topographic mapping in the mammalian visual system. Neuron 20:235-243.

Gale NW, Holland SJ, Valenzuela DM, Flenniken A, Pan L, Ryan TE, Henkemeyer M, Strebhardt K, Hirai H, Wilkinson DG, Pawson T, Davis S, Yancopoulos GD (1996) Eph receptors and ligands comprise two major specificity subclasses and are reciprocally compartmentalized during embryogenesis. Neuron 17:9-19.

Gao PP, Zhang JH, Yokoyama M, Racey B, Dreyfus CF, Black IB, Zhou R (1996) Regulation of topographic projection in the brain: Elf-1 in the hippocamposeptal system. Proc Natl Acad Sci U S A 93:11161-11166.

Gierer A (1988) Spatial organization and genetic information in brain development. Biol Cybern 59:13-21.

Goodhill G, Xu J (2005) The development of retinotectal maps: a review of models based on molecular gradients. Network 16:5-34.

Goodhill GJ (2007) Contributions of theoretical modeling to the understanding of neural map development. Neuron 56:301-311.

Goodhill GJ, Richards LJ (1999) Retinotectal maps: molecules, models and misplaced data. Trends Neurosci 22:529-534.

Helmbacher F, Schneider-Maunoury S, Topilko P, Tiret L, Charnay P (2000) Targeting of the EphA4 tyrosine kinase receptor affects dorsal/ventral pathfinding of limb motor axons. Development 127:3313-3324.

Honda H (2003) Competition between retinal ganglion axons for targets under the servomechanism model explains abnormal retinocollicular projection of Eph receptor-overexpressing or ephrin-lacking mice. J Neurosci 23:10368-10377.

Jeon CJ, Strettoi E, Masland RH (1998) The major cell populations of the mouse retina. J Neurosci 18:8936-8946.

Kaas JH (1997) Topographic maps are fundamental to sensory processing. Brain Res Bull 44:107-112.

Kania A, Jessell TM (2003) Topographic motor projections in the limb imposed by LIM homeodomain protein regulation of ephrin-A:EphA interactions. Neuron 38:581-596.

Koulakov AA, Tsigankov DN (2004) A stochastic model for retinocollicular map development. BMC Neurosci 5:30.

Law MI, Constantine-Paton M (1980) Right and left eye bands in frogs with unilateral tectal ablations. Proc Natl Acad Sci U S A 77:2314-2318.

Lemke G, Reber M (2005) Retinotectal mapping: new insights from molecular genetics. Annu Rev Cell Dev Biol 21:551-580.

Luo L, Flanagan JG (2007) Development of continuous and discrete neural maps. Neuron 56:284-300.

Marcus RC, Matthews GA, Gale NW, Yancopoulos GD, Mason CA (2000) Axon guidance in the mouse optic chiasm: retinal neurite inhibition by ephrin "A"-expressing hypothalamic cells in vitro. Dev Biol 221:132-147.

McLaughlin T, O'Leary DD (2005) Molecular gradients and development of retinotopic maps. Annu Rev Neurosci 28:327-355.

McLaughlin T, Torborg CL, Feller MB, O’Leary DD (2003) Retinotopic map refinement requires spontaneous retinal waves during a brief critical period of development. Neuron 40:1147-1160.

Monschau B, Kremoser C, Ohta K, Tanaka H, Kaneko T, Yamada T, Handwerker C, Hornberger MR, Löschinger J, Pasquale EB, Siever DA, Verderame MF, Müller BK, Bonhoeffer F, Drescher U (1997) Shared and distinct functions of RAGS and ELF-1 in guiding retinal axons. EMBO J 16:1258-1267.

Mrsic-Flogel TD, Hofer SB, Creutzfeldt C, Cloëz-Tayarani I, Changeux JP, Bonhoeffer T, Hübener M (2005) Altered map of visual space in the superior colliculus of mice lacking early retinal waves. J Neurosci 25:6921-6928.

O’Leary DD, Wilkinson DG (1999) Eph receptors and ephrins in neural development. Curr Opin Neurobiol 9:65-73.

Pak W, Hindges R, Lim YS, Pfaff SL, O'Leary DD (2004) Magnitude of binocular vision controlled by islet-2 repression of a genetic program that specifies laterality of retinal axon pathfinding. Cell 119:567-578. 
Palmer A, Klein R (2003) Multiple roles of ephrins in morphogenesis, neuronal networking, and brain function. Genes Dev 17:1429-1450.

Pasquale EB (2005) Eph receptor signalling casts a wide net on cell behaviour. Nat Rev Mol Cell Biol 6:462-475.

Prestige MC, Willshaw DJ (1975) On a role for competition in the formation of patterned neural connexions. Proc R Soc Lond B Biol Sci 190:77-98.

Reber M, Burrola P, Lemke G (2004) A relative signalling model for the formation of a topographic neural map. Nature 431:847-853.

Schmidt JT, Easter SS (1978) Independent biaxial reorganization of the retinotectal projection: a reassessment. Exp Brain Res 31:155-162.

Sharma SC (1972) Redistribution of visual projections in altered optic tecta of adult goldfish. Proc Natl Acad Sci U S A 69:2637-2639.
Simpson HD, Goodhill GJ (2011) A simple model can unify a broad range of phenomena in retinotectal map development. Biol Cybern 104:9-29.

Sperry RW (1963) Chemoaffinity in the orderly growth of nerve fiber patterns and connections. Proc Natl Acad Sci U S A 50:703-710.

Triplett JW, Owens MT, Yamada J, Lemke G, Cang J, Stryker MP, Feldheim DA (2009) Retinal input instructs alignment of visual topographic maps. Cell 139:175-185.

Turing A (1952) The chemical basis of morphogenesis. Philos Trans R Soc Lond Ser B Biol Sci 237:37-72.

Willshaw D (2006) Analysis of mouse EphA knockins and knockouts suggests that retinal axons programme target cells to form ordered retinotopic maps. Development 133:2705-2717. 